\title{
Molecular structures of thymidine isomers isolated in low-temperature inert matrices
}

\author{
A.Yu. Ivanov ${ }^{1}$, S.A. Krasnokutski ${ }^{2}$, and G.G. Sheina ${ }^{1}$ \\ ${ }^{1}$ B. Verkin Institute for Low Temperature Physics and Engineering of the National Academy of \\ Sciences of Ukraine, 47 Lenin Ave., Kharkov 61103, Ukraine \\ E-mail: ivanov@ilt.kharkov.ua \\ ${ }^{2}$ Max-Planck-Institut für Strömungsforschung, Bunsenstraße 10, Göttingen 37073, Germany
}

The Fourier transform infrared spectra of $2^{\prime}$-deoxyribonucleoside - thymidine $(\mathrm{dT})$ in low-temperature Ar matrices are obtained in the range $4000-1300 \mathrm{~cm}^{-1}$. It is determined that anti-conformers of thymidine are dominant. The ribose rings of the main anti-conformers dT_a0, $\mathrm{dT}$-a1 are in the $\mathrm{C} 2$ '-endo conformation, but the ribose rings of minor anti-conformers $\mathrm{dT}$-a2, dT_a3 have the $\mathrm{C}^{\prime}$-endo conformation, stabilized by intramolecular hydrogen bonds $\mathrm{O}^{\prime} \mathrm{H}_{\text {....O }} 5^{\prime}$ and $\mathrm{O}^{\prime} \mathrm{H} . . . \mathrm{O} 3^{\prime}$, respectively. The main syn-conformer dT_s2 is stabilized by the intramolecular hydrogen bond $\mathrm{O}^{\prime} \mathrm{H} \ldots \mathrm{O} 2$ and has $\mathrm{C}^{\prime}$-endo conformation of the ribose ring.

PACS: 33.15.-e, 67.80.Cx

The structural components of DNA - nucleosides and their derivatives - are important objects of investigation for the modern science of life [1-11]. The main experimental methods of investigation of nucleosides are NMR spectroscopy and crystallography [1]. But the results of investigations by these methods depend strongly on the intermolecular interactions. For example, only one form of several possible conformers can be stabilized in crystals [1]. Owing to competing interactions with the solvent, NMR spectroscopy gives no way to obtain direct data about intramolecular hydrogen bonding [4,5]. These limitations are absent in the method of matrix isolation, where molecular isomers from the gas phase are trapped in low-temperature inert matrices [12]. We previously used the Fourier transform infrared (FTIR) matrix isolation spectroscopy for the first time in the investigation of pyrimidine nucleosides isolated in low-temperature inert matrices $[10,11]$. The evaporation of uridine and $\mathrm{dT}$ without thermodestruction was demonstrated, and the intramolecular $\mathrm{H}$-bond $\mathrm{O}^{\prime} \mathrm{H} . . . \mathrm{O} 2$ was detected $[10,11]$. In the present research, FTIR spectra of dT in Ar matrices were obtained by using an enhanced experimental setup. The new spectral data suggest the existence of more types of isomers with intramolecular $\mathrm{H}$-bonds in the isolated pyrimidine nucleosides than had been considered before [7-11].

\section{Experimental and computational methods}

The basic features of the FTIR spectrometer have been described previously [11,13-15]. For this paper the FTIR spectra of thymidine and the auxiliary substance 1-methyl-thymine were obtained in the ranges 4000-1300 $\mathrm{cm}^{-1}$ with a $\mathrm{CaF}_{2}$ beamsplitter at an apodized resolution of $0.4 \mathrm{~cm}^{-1}$. The matrix isolation

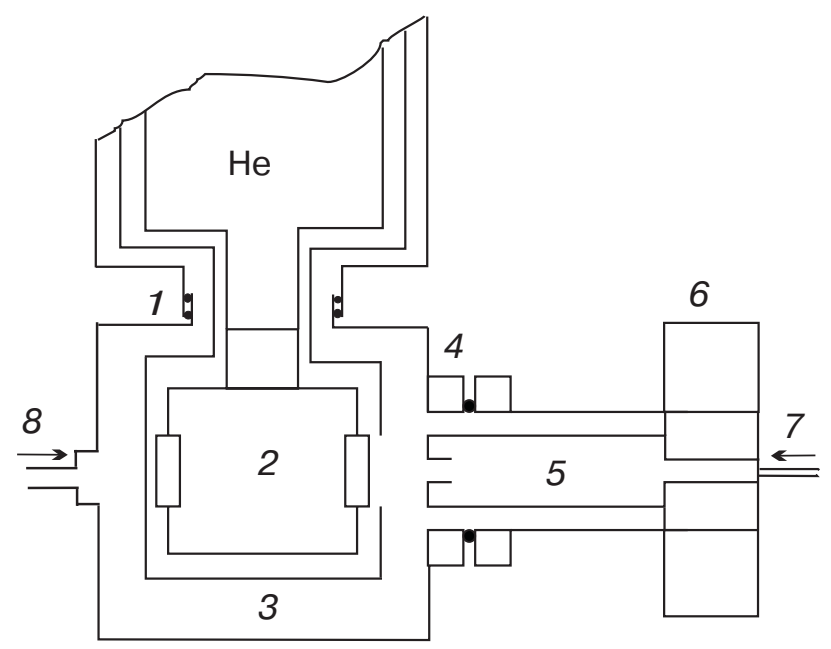

Fig. 1. The general scheme of low-temperature setup based on liquid He cryostat: rotating vacuum seal (1), cryogenic block with cold mirrors and QCM (2), rotating nitrogen shield (3), flange with indium seal (4), Knudsen cell (5), electric heater of Knudsen cell (6), Ar flow through Knudsen cell (7), outside Ar flow (8). 
setup was based on a liquid helium cryostat with a nitrogen shield (Fig. 1). Two low-temperature differential quartz crystal microbalances (QCM) and two metal mirrors were placed on the copper holder in the vacuum chamber (Fig. 1) and had a working temperature in the range $5-40 \mathrm{~K}$. The QCM was used for the measurements of the absolute intensity of the molecular beams and the matrix-to-sample ratio $(\mathrm{M} / \mathrm{S})$ [13]. Owing to QCM, we have the capability of working not only with an Ar flux passing through the Knudsen cell but with an outside flux of cold Ar gas also (Fig. 1).

All nucleosides are very thermally labile molecules, and for their evaporation a special Knudsen cell with reduced molecular beam losses was constructed. As is shown in Fig. 2, the geometry of the Knudsen cell and its disposition to low-temperature mirror are very important for effective operation. The data in Fig. 2 were obtained by the statistical Monte Carlo method, which is very useful for the simulation of complicated vacuum systems [16]. This evaporation cell is characterized by a working vapor pressure of around $10^{-5}$ Torr and Knudsen number of over 100 . This evaporation cell is more effective by a factor of more than 500-1000 over one of the cells used in our previous experiments with simple compounds [13-15]. In comparison with our previous work [11], for the present experiments the distance $L$ (Fig. 2) was decreased from $4.5 R$ to $2.5 R$. The typical intensities of molecular beams of thymidine were about $40-70 \mathrm{ng} /\left(\mathrm{s} \cdot \mathrm{cm}^{2}\right)$ at evaporation temperatures $410-430 \mathrm{~K}$ without any thermodestruction. Thymidine (commercial substance from Sigma) was used without additional purifica-

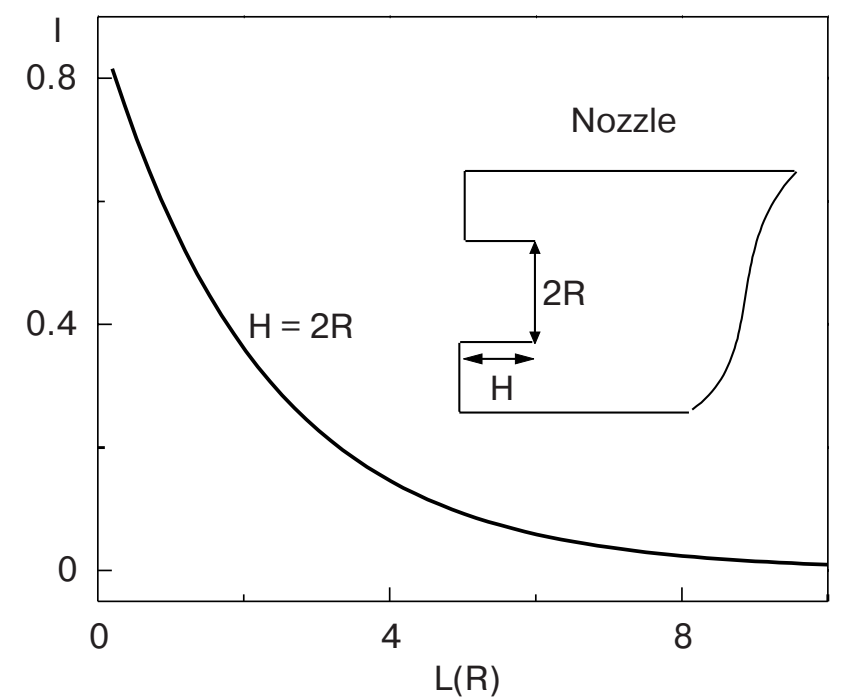

Fig. 2. The relationship of the efficiency of evaporation cell $(I)$ as function of the distances $L$ between cell and cold mirror. $L$ is in units $R, R$ is the radius of outlet nozzle of Knudsen cell. tion. The auxiliary substance 1-methyl-thymine was synthesized at the Kharkov National University (Kharkov, Ukraine). All substances were annealed to remove impurities such as sorbed $\mathrm{H}_{2} \mathrm{O}, \mathrm{CO}_{2}$, and $\mathrm{N}_{2}$ in the initial phase of evaporation. The inert gas Ar was more than $99.99 \%$ pure and deposited on the mirrors at $11 \mathrm{~K}$. To improve the optical characteristics of the Ar matrices, before the deposition of matrix samples a thin layer of pure Ar was deposited on the mirrors over a temperature range of 35-20 K [15]. The quantum-chemical ab initio calculations of the relative energies and vibrational spectra of thymidine conformers were performed by the program PC GAMESS version 6.0 [17] of the GAMESS (US) QC package [18].

\section{Results and discussion}

The peculiarities of vibrational spectra of $d T$ conformers. As is evident from Fig. 3, in the region of the stretching vibrations $v(\mathrm{OH}), v(\mathrm{NH})$, the frequency of the $v(\mathrm{~N} 3 \mathrm{H})$ stretching vibration of $\mathrm{dT}$ coincides well with $v(\mathrm{~N} 3 \mathrm{H})$ of 1-methyl-thymine. Because of this, four absorption bands in this region (Fig. 3) can belong to two $\mathrm{OH}$ groups of $2^{\prime}$-deoxyribose $-\mathrm{O}^{\prime} \mathrm{H}$, $\mathrm{O}^{\prime} \mathrm{H}$ only (Fig. 3). The magnification of the number of bands can be explained by intramolecular hydrogen bonds in the conformational structures, which were considered in this paper (Fig. 4). It is known that the conformational-flexible ribose rings of nucleosides has a puckered structure and $\mathrm{C}^{\prime}$-endo, $\mathrm{C}^{\prime}$ '-endo are the

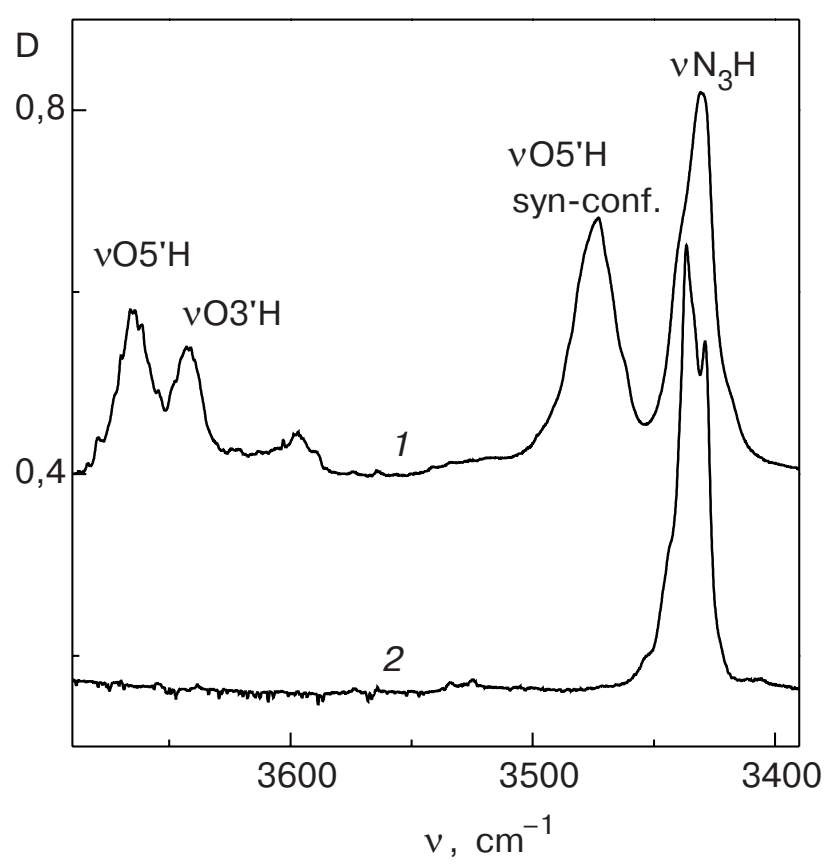

Fig. 3. The FTIR spectra of thymidine (1) and 1-methylthymine (2) isolated in $\operatorname{Ar}$ matrices $(T=12 \mathrm{~K}$, $M / S=700)$ in the $\mathrm{O}-\mathrm{H}, \mathrm{N}-\mathrm{H}$ stretching region $\left(3690-3390 \mathrm{~cm}^{-1}\right)$. 

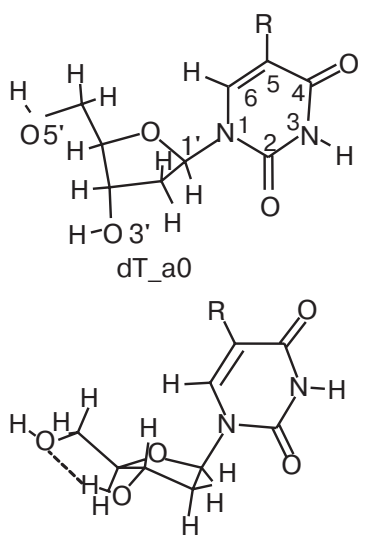

dT_a2<smiles></smiles>

dT_s1

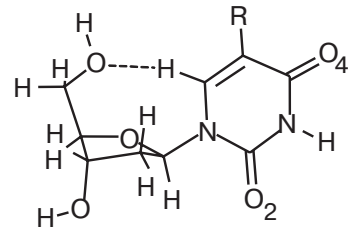

dT_a1
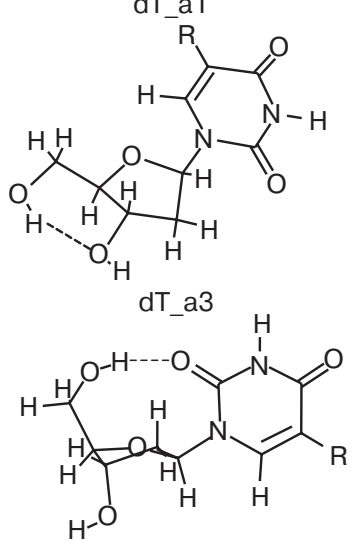

dT_s2

Fig. 4. The conformational structures of thymidine which are stable at the different level of calculation $(\mathrm{HF} / 3-21 \mathrm{G}(p), \quad \mathrm{HF} / 6-31 \mathrm{G}(d, p) \quad$ and $\mathrm{MP} 2 / 6-31 \mathrm{G}(d, p))$. Intramolecular $\mathrm{H}$-bonds are represented with dashed lines. The symbol $\mathrm{R}$ - represents a $\mathrm{CH}_{3}$ group.

main equilibrium conformations of the ribose ring in solutions [1]. As is indicated in Fig. 4, the transition $\mathrm{C} 3$ '-endo $\rightarrow \mathrm{C} 2$ '-endo has no effect on the structure of the H-bonds in the syn-conformers dT_s1, dT_s2 (Fig. 4). In the anti-conformers dT_a2, dT_a3 the transition $\mathrm{C} 2^{\prime}$-endo $\rightarrow \mathrm{C}^{\prime}$ '-endo leads to the formation

of the H-bonds $\mathrm{O3}^{\prime} \mathrm{H} . . . \mathrm{O}^{\prime}$, O5'H...O3' (Fig. 4). The choice of the structure dT_a1 with the orientation of hydroxymethyl group gauche ${ }^{+}$and torsion angle $\gamma$ $\left(<\mathrm{C} 3^{\prime}-\mathrm{C}^{\prime}-\mathrm{C}^{\prime} \mathrm{O}^{\prime}\right)=+50^{\circ}$ are close to the structures with hydrogen bonds $\mathrm{C} 6 \mathrm{H}$... O $5^{\prime}$ in the anti-conformers of uridine and cytosine $[7,8]$. The structure dT_a0 with the orientation of hydroxymethyl group gauche $-\left(<\gamma \approx-65^{\circ}\right)$ and the lack of any classical linear H-bonds (Fig. 4) were considered also. The stabilization of the position of the pyrimidine ring in the dT_a0, dT_a2, dT_a3 conformation can be effected by the electrostatic interaction between atoms: $\mathrm{C} 6 \mathrm{H} \longleftrightarrow$ $\mathrm{O}^{\prime}{ }^{\prime}$ and $\mathrm{C} 2 \mathrm{O} \longleftrightarrow \mathrm{H}^{\prime}$. The orientation of the methyl group with respect to the carbonyl group $\mathrm{C} 2 \mathrm{O}$ are close to the structure A from [19], where two hydrogen atoms positioned above and below N1-C2-O plane (Fig. 4). All conformers from Fig. 4 may be considered as local minima, since they were stable at the $\mathrm{HF} / 3-21 \mathrm{G}(p), \mathrm{HF} / 6-31 \mathrm{G}(d, p)$, and $\mathrm{MP} 2 / 6-31 \mathrm{G}(d, p)$ levels of calculation and have no imaginary frequencies in the calculated spectra.

The experimental and calculated spectra are compared in the region of $v(\mathrm{OH}), v(\mathrm{NH}), v(\mathrm{CH})\left(\mathrm{Ta}^{-}\right.$ ble 1). From this table we notice that the calculated frequencies of the free $\mathrm{O}^{\prime} \mathrm{H}, \mathrm{O}^{\prime} \mathrm{H}$, and $\mathrm{N} 3 \mathrm{H}$ groups are in good agreement with the experimental frequencies for all conformers. The band $3482 \mathrm{~cm}^{-1}$ (Fig. 3, Table 1) can be assigned to the H-bonded vibration O5'H...O2 in the syn-conformations. According to our calculations, the vibration $v\left(h_{b} \mathrm{O}^{\prime} \mathrm{H}\right)$ in conformer dT_s2 has a frequency about $40 \mathrm{~cm}^{-1}$ lower than in the dT_s1 conformer (Table 1). We can see in our experimental spectra that the band at $3482 \mathrm{~cm}^{-1}$ does not

Table 1

The parameters of experimental FTIR spectra in Ar matrices and calculated by the method 6-31G $(d, p)$ spectral bands of thymidine conformers in the $3700-3000 \mathrm{~cm}^{-1}$ region

\begin{tabular}{|c|c|c|c|c|c|c|c|c|c|c|c|c|c|c|}
\hline \multirow{2}{*}{$\begin{array}{l}\text { Conformer } \\
\text { mode }\end{array}$} & \multicolumn{2}{|c|}{ Experiment } & \multicolumn{2}{|c|}{ dT_a0 } & \multicolumn{2}{|c|}{ dT_a1 } & \multicolumn{2}{|c|}{ dT_a2 } & \multicolumn{2}{|c|}{ dT_a3 } & \multicolumn{2}{|c|}{ dT_s1 } & \multicolumn{2}{|c|}{ dT_s2 } \\
\hline & $v, \mathrm{~cm}^{-1}$ & $I$ & $v, \mathrm{~cm}^{-1}$ & $I^{a}$ & $v, \mathrm{~cm}^{-1}$ & $I^{a}$ & $v, \mathrm{~cm}^{-1}$ & $I^{a}$ & $v, \mathrm{~cm}^{-1}$ & $I^{a}$ & $v, \mathrm{~cm}^{-1}$ & $I^{a}$ & $v, \mathrm{~cm}^{-1}$ & $I^{a}$ \\
\hline vO5'H & 3665 & 3.1 & 3673 & 76 & 3367 & 72 & 3673 & 84 & & & & & & \\
\hline vO3'H & 3641 & 1.8 & 3654 & 64 & 3659 & 54 & & & 3659 & 65 & 3681 & 76 & 3660 & 54 \\
\hline$v$ hb_O3'H & $3620^{*}$ & $0.8^{*}$ & & & & & 3638 & 107 & & & & & & \\
\hline$v$ hb_O5'H & $3597^{*}$ & $0.5^{*}$ & & & & & & & 3639 & 86 & & & & \\
\hline$v$ hb_O5'H & 3482 & 6.2 & & & & & & & & & 3626 & 161 & 3589 & 351 \\
\hline$v \mathrm{~N} 3 \mathrm{H}$ & 3428 & 9.2 & 3439 & 106 & 3441 & 103 & 3438 & 106 & 3438 & 106 & 3435 & 103 & 3436 & 106 \\
\hline vC6H & 3075 & 0.5 & 3059 & 4 & 3043 & 14 & 3071 & 7 & 3070 & 8 & 3022 & 6 & 3026 & 5 \\
\hline
\end{tabular}

C o $\mathrm{m} \mathrm{me} \mathrm{n} \mathrm{t:} I$ - relative integral intensities. $I^{a}$ - absolute integral intensities $(\mathrm{km} / \mathrm{mol})$. vhb_OH - bands of groups involved in the intramolecular H-bonds. * - after deconvolution of the wide band $3598 \mathrm{~cm}^{-1}$ on the Gaussian contours. 


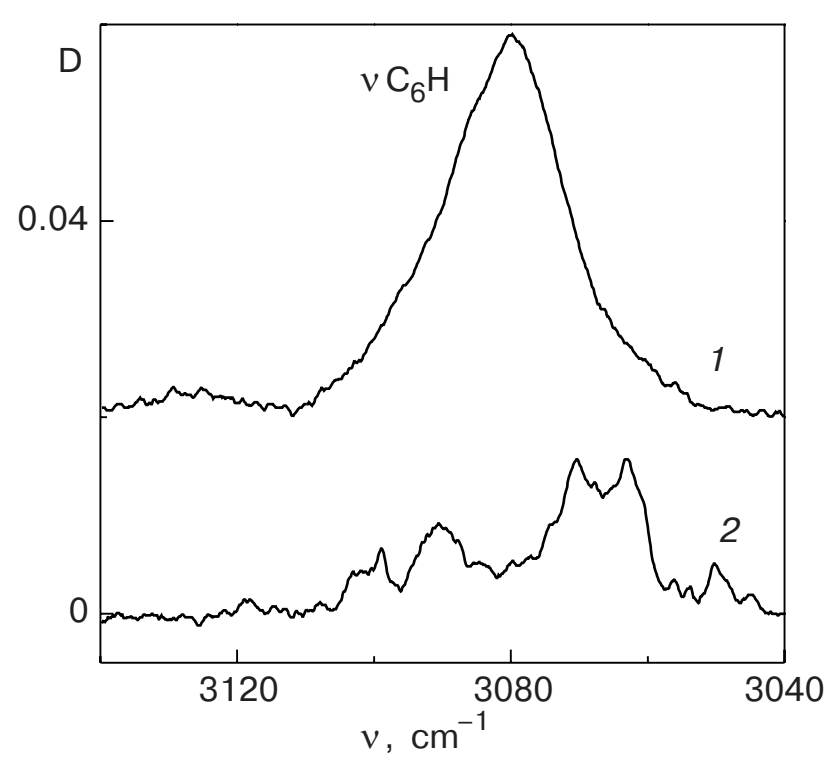

Fig. 5. The FTIR spectra of thymidine (1) and 1-methylthymine (2) isolated in $\operatorname{Ar}$ matrices $(T=12 \mathrm{~K}$, $M / S=700)$ in the $\mathrm{C} 5-\mathrm{H}, \mathrm{C} 6-\mathrm{H}$ stretching region $\left(3140-3040 \mathrm{~cm}^{-1}\right)$.

have a high-frequency shoulder in Ar matrices (Fig. 3) and the occupancy of conformer dT_s1 can be neglected. The calculated frequencies and intensities of the characteristic bands of the conformers dT_a2 and $\mathrm{dT} \_\mathrm{a} 3$ are in close agreement (Table 1). This result is supported by the experiment in Ar matrices, where we can see only the one wide band $3598 \mathrm{~cm}^{-1}$ with a high-frequency shoulder (Fig. 3). Contrary to the calculated parameters of $v \mathrm{C} 6 \mathrm{H}$ (Table 1), only one band of $v \mathrm{C} 6 \mathrm{H}$ vibration was detected in the experimental spectrum (Fig. 5). The influence of the intramolecular hydrogen bonds $\mathrm{C} 6 \mathrm{H} . . . \mathrm{O}^{\prime}$ in conformer $\mathrm{dT}$-a1 on the $v \mathrm{C} 6 \mathrm{H}$ parameters was not detected experimentally, as the free $\mathrm{C} 6 \mathrm{H}$ group in 1-methyl-thymine has Fermi resonance splitting in the spectra (Fig. 5). Consequently, it may be considered that the anti-conformers $\mathrm{dT} \_\mathrm{a} 0$ and $\mathrm{dT}$ _a1 are practically indistinguishable in the investigated region.

The populations of $d T$ conformers in the low-temperature matrices. It is known that the conformers' occupancies in matrices may differ widely from those in the gas phase. The effect of interconversion is observed at low barriers between conformational isomers [12]. We tested the interconversion by using the annealing of matrix samples. The annealing of matrix samples at $30 \mathrm{~K}$ has no influence on the conformational equilibrium. It follows that the barrier heights of dT conformers $>2.5-3 \mathrm{kcal} /$ mole and their conformational equilibrium in the gas phase at the evaporation temperature must be close to equilibrium in Ar matrices at $12 \mathrm{~K}$. For comparison with the real experimental data the relative free Gibbs energy $\Delta G$ was estimated by the standard method [20]:

$$
\Delta G_{A B}(T)=\Delta E+\Delta Z P E+\int_{0}^{T} \Delta C d T-T \Delta S(T) .
$$

The relative electronic energy $\Delta E$ was estimated at the MP2/6-31G $(d, p)$ level of ab initio calculation, and the relative zero-point vibrational energy $\triangle Z P E$ and temperature-dependent contributions of rotation and vibration were estimated at the $\mathrm{HF} / 6-31 \mathrm{G}(d, p)$ level for temperatures of 298 and $420 \mathrm{~K}$ (Table 2).

The association of $\Delta G$ and the experimental spectral data can be expressed by the standard equation:

$$
\Delta G_{A B}(T)=-R T \ln K_{A B}=-R T \ln \left(\eta_{A} / \eta_{B}\right),
$$

where $K_{A, B}$ is the equilibrium constant of conformers $A, B$, and $\eta_{A}, \eta_{B}$ are the populations of these conformers.

To define the equilibrium constants by using the experimental spectra it is necessary to know the molar extinction coefficients of the characteristic spectral bands or their ratios. The ratios of molar extinction coefficients of characteristic bands may be determined through the redistribution of the intensities of the characteristic bands under the influence of changing evaporation temperature or through the UV irradiation of matrix samples. If the corresponding experiments are difficult, the $a b$ initio calculations of the intensities of the characteristic spectral bands may be used. Ab initio calculations usually overestimate the absolute infrared intensity of vibrational bands $[21,22]$, but the ratio of the experimental and calculated intensities $I$ coincides much better. The equilibrium constant of isomers $a$ and $b$ has been presented as $[23,24]$ :

$$
K_{A B}=\left(\frac{\sum I_{a(\exp )}}{\sum I_{a(\text { calc })}}\right)\left(\frac{\sum I_{b(\text { calc })}}{\sum I_{b(\exp )}}\right) .
$$

Unlike some studies [23,24], we have used Eq. (1) with the intensities of the $v(\mathrm{OH}), v(\mathrm{NH})$, and $v(\mathrm{CH})$ stretching vibrations only. With our data the best agreement between the calculated and experimental results is observed for the ratio of the intensities of stretching vibrations. For determination of $K_{A B}$ different combinations of the experimental and calculated intensities from Table 1 were used. As discussed above, the anti-conformers in the pairs: dT_a0 and dT_a1, dT_a2 and dT_a3 are practically indistinguishable in the matrix spectra. Therefore, for the sake of simplicity, the conformer dT_a0 was ignored.

According to the relative electronic energy calculations, the syn-conformer dT_s2 with the 
Calculated and experimental relative energies $(\mathrm{kcal} / \mathrm{mole})$ of the thymidine conformers in the isolated state

\begin{tabular}{|c|c|c|c|c|c|c|}
\hline $\begin{array}{c}\text { Conformer } \\
\text { method }\end{array}$ & dT_a0 & dT_a1 & dT_a2 & dT_a3 & dT_s1 & dT_s2 \\
\hline $\mathrm{HF} / 3-21 \mathrm{G}(p)$ & 5.4 & 2.4 & 2.6 & 4.6 & 3.3 & $\begin{array}{c}0 \\
(-865.3452)^{*}\end{array}$ \\
\hline $\mathrm{HF} / 6-31 \mathrm{G}(d, p)$ & 2.9 & 1.6 & 1.8 & 3.4 & 1.5 & $\begin{array}{c}0 \\
(-870.1091)^{*}\end{array}$ \\
\hline $\mathrm{MP} 2 / 6-31 \mathrm{G}(d, p)$ & 5.7 & 3.0 & 3.8 & 4.9 & 2.6 & $\begin{array}{c}0 \\
(-872.6776) *\end{array}$ \\
\hline $\begin{array}{c}\Delta \mathrm{G}(298 \mathrm{~K})^{* *} \\
\mathrm{MP} 2 / 6-31 \mathrm{G}(d, p)\end{array}$ & 3.3 & 1.8 & 2.1 & 3.4 & 1.6 & 0 \\
\hline $\begin{array}{c}\Delta \mathrm{G}(420 \mathrm{~K})^{* *} \\
\mathrm{MP} 2 / 6-31 \mathrm{G}(d, p)\end{array}$ & 2.4 & 1.4 & 1.6 & 2.9 & 1.2 & 0 \\
\hline $\begin{array}{l}\Delta \mathrm{G}(420 \mathrm{~K}) \\
\text { Experiment }\end{array}$ & - & 0 & 1.8 & 2.2 & - & 1.1 \\
\hline
\end{tabular}

* - absolute energies in a.u. are indicate in brackets. ** $\mathrm{HF} / 6-31 \mathrm{G}(d, p)$ level.

intramolecular hydrogen bond $\mathrm{O}^{\prime} \mathrm{H} . . \mathrm{O} 2$ and conformation of the sugar ring - $\mathrm{C} 2$ '-endo has the minimal energy among all conformers at all levels of optimization (Table 2). The data in Table 2 demonstrate disagreement between the experimental and calculated occupancies of the dT_a1 and dT_s2 conformers, but the experimental and calculated data coincide well for the minor anti-conformers dT_a2 and dT_a3 (Table 2). The influence of the vibrational-rotational contribution to the free energy reduces the difference of conformers' energies significantly, and this is especially noticeable with increasing evaporation temperature (Table 2). Because of this, conformers dT_s2 may have an essential effect on biological processes at the relatively low physiological temperatures.

\section{Conclusions}

It was shown that FTIR matrix isolation spectroscopy is a helpful method for the investigation of the molecular structure of nucleoside conformational isomers. It was established that at evaporation temperatures of up to $430 \mathrm{~K}$ thymidine may be evaporated for an appreciable length of time and trapped in inert matrices without any thermodestruction. Anti-conformers of thymidine are dominant in the isolated state. The main anti-conformer dT_a1, has the $\mathrm{C} 2{ }^{\prime}$-endo conformation of the ribose ring. The minor anti-conformers dT_a2, dT_a3 have the C3'-endo conformation of the ribose ring, stabilized by intramolecular hydrogen bonds O3'H...O5' and O5'H...O3' respectively. The intramolecular hydrogen bonds $\mathrm{O}^{\prime} \mathrm{H} . . . \mathrm{O} 5^{\prime}$, and O5'H...O3' may be regarded as an indicator of the transition $\mathrm{C} 2^{\prime}$-endo $\rightarrow \mathrm{C}^{\prime}$-endo between conforma- tions of ribose ring in the anti-conformers of thymidine. The thymidine dT_s2 syn-conformer is stabilized by the intramolecular hydrogen bond O5'H...O2 and the dominant conformation of ribose ring is $\mathrm{C}^{\prime}$-endo.

\section{Acknowledgement}

This investigation was supported by the Ukrainian Academy of Sciences and in part by the INTAS-International Association under grant No. INTAS 00-00911.

1. W. Saenger, Principles of Nucleic Acid Structure, Springer-Verlag, New York (1984).

2. G.A. Jeffrey and W. Saenger, Hydrogen Bonding in Biological Structures, Springer-Verlag, Berlin (1991).

3. S.N. Rao, Nucleosides and Nucleotides 14, 1179 (1995)

4. F. Seela, H. Debelak, H. Reuter, G. Kastner, and I.A. Mikhailopulo, Tetrahedron 55, 1295 (1999).

5. J.J. Barchi Jr., L.-S. Jeong, M.A. Siddiqui, V.E. Marquez, and J. Biochem, Biophys. Methods 34, 11 (1997).

6. J.M. Gavira, M. Campos, G. Diaz, A. Hernanz, and R. Navarro, Vibrational Spectroscopy 15, 1 (1997).

7. N. Leulliot, M. Ghomi, G. Scalmani, and G. Berthier, J. Phys. Chem. A103, 8716 (1999).

8. N. Leulliot, M. Ghomi, H. Jobic, O. Bouloussa, V. Baumruk, and C. Coulombeau, J. Phys. Chem. B103, 10934 (1999).

9. O.V. Shishkin, A. Pelmenschikov, D.M. Hovorun, and J. Leszczynski, J. Mol. Struct. 526, 329 (2000).

10. S.A. Krasnokutski, A.Yu. Ivanov, V. Izvekov, G.G. Sheina, and Yu.P. Blagoi, XXIV European Congress 
on Molecular Spectroscopy, Prague, Czech Repablic, 470 (1998).

11. S.A. Krasnokutski, A.Yu. Ivanov, V. Izvekov, G.G. Sheina, and Yu.P. Blagoi, J. Mol. Struct. 482-483, 249 (1998).

12. A.J. Barnes, J. Mol. Struct. 113, 161 (1984).

13. A.Yu. Ivanov, A.M. Plokhotnichenko, E.D. Radchenko, G.G. Sheina, and Yu.P. Blagoi, J. Mol. Struct. 372, 91 (1995).

14. A.Yu. Ivanov, A.M. Plokhotnichenko, V. Izvekov, G.G. Sheina, and Yu.P. Blagoi, J. Mol. Struct. 408-409, 459 (1997).

15. A.Yu. Ivanov, G. Sheina, and Yu.P. Blagoi, Spectrochim. Acta A55, 219 (1999).

16. G.L. Saksagansky, Molecular Beams in the Composite Vacuum Structures, Atomizdat, Moscow (1980) (in Russian ).

17. Alex.A. Granovsky, http://classic.chem.msu.su/gran /gamess/index.html.
18. M.W. Schmidt, K.K. Baldridge, J.A. Boatz, S.T. Elbert, M.S. Gordon, J.J. Jensen, S. Koseki, N. Matsunaga, K.A. Nguyen, S. Su, T.L. Windus, M. Dupuis, and J.A. Montgomery, J. Comput. Chem. 14, 1347 (1993).

19. M.J. Nowak, L. Lapinski, D.C. Bienko, and D. Michalska, Spectrochim. Acta A53, 855 (1997).

20. J.S. Kwiatkowski and J. Leszczynsky, Chem. Phys. Lett. 204, 430 (1993).

21. M.J. Nowak, L. Les, and L. Adamowicz, Trends Phys. Chem. 4, 137 (1994).

22. Y. Yamaguchi, M. Frisch, J. Gaw, H.F. Schaefer III, and J.S. Binkley, J. Chem. Phys. 84, 2262 (1986).

23. M.J. Nowak, L. Lapinski, and J. Fulara, Spectrochim. Acta 45A, 229 (1989).

24. H. Vranken, J. Smets, G. Maes, L. Lapinski, M.J. Nowak, and L. Adamovicz, Spectrochim. Acta 50A, 875 (1994). 